\title{
Complex Analysis for Reconstruction from Controlled Motion
}

\author{
R. Andrew Hicks David Pettey Kostas Daniilidis Ruzena Bajcsy \\ GRASP Laboratory, \\ Department of Computer and Information Science \\ University of Pennsylvania \\ University of Pennsylvania, Philadelphia PA 19104, USA, \\ Telephone: (215) 898-0351 \\ Fax: (215) 573-2048 \\ rah@grip.cis. upenn.edu, \\ WWW home page: http://www.cis.upenn.edu/ rah
}

\begin{abstract}
We address the problem of control-based recovery of robot pose and the environmental lay-out. Panoramic sensors provide us with a $1 D$ projection of characteristic features of a $2 D$ operation map. Trajectories of these projections contain information about the position of a priori unknown landmarks in the environment. We introduce here the notion of spatiotemporal signatures of projection trajectories. These signatures are global measures, characterized by considerably higher robustness with respect to noise and outliers than the commonly applied point correspondence. By modeling the $2 D$ motion plane as the complex plane we show that by means of complex analysis the reconstruction problem is reduced to a system of two quadratic - or even linear in some cases - equations in two variables. The algorithm is tested in simulations and real experiments.
\end{abstract}

Keywords: Panoramic sensor, reconstruction, residue. 


\section{Introduction}

Consider the following situation. We are given two points in the plane $z_{1}$ and $z_{2}$ and a sensor that can measure the angle between $z_{1}$ and $z_{2}$ from any position in the plane at which it is placed. If the sensor undergoes a circular motion (figure 1) then we may record the angles in a list and then plot each angle against its index in the list. For example, in the right of figure 1 we see such a graph for two points.
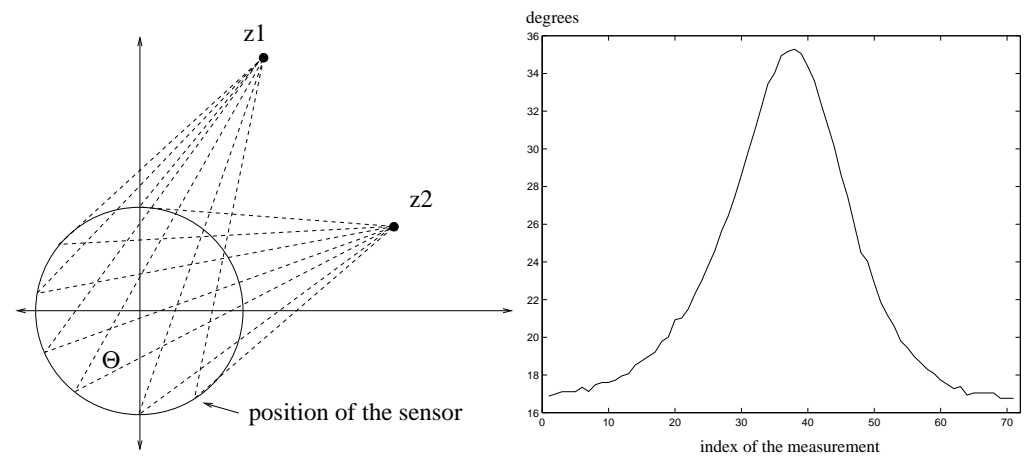

Figure1. The problem: find the values of $z_{1}$ and $z_{2}$ by moving on a circle and measuring the angle $\Theta$ during the motion. On the left we see the actual motion of the sensor, while on the right we see a plot of the measured angles between $z_{1}$ and $z_{2}$ vs. the index of the measurement in the list of all measurements. This data is taken from a real experiment.

The primary motivation for this paper is to investigate the extent to which the data displayed on the right in figure 1 characterizes the two unknown points. In other words, we want to solve the inverse problem: given this data, how can the scene be reconstructed? In the world, of course, there is no natural Cartesian frame, so what we really solve for is the magnitudes of $z_{1}$ and $z_{2}$, and the angle between them with respect to the center of the circular motion. Throughout this paper the scale will always be determined by taking the radius of the circle to be our unit.

\section{$1.1 \quad$ Signatures}

In many vision problems one must obtain geometric information about the environment and to do this a great deal of data is recorded. This data can be thought of as a long list of numbers, i.e. we measure a vector $\mathbf{c}=\left(c_{1}, c_{2}, \ldots, c_{n}\right)$. Then one seeks to solve a system of equations $F_{j}(\mathbf{c}, \mathbf{z})=\mathbf{0}, j=1, \ldots, m$, where the unknown is $\mathbf{z}=\left(z_{1}, z_{2}, \ldots, z_{k}\right)$, and $z_{i} \in \mathbf{R}$ or $\mathbf{C}$. Generally $k, m$ and $n$ are large integers and the system is non-linear and over-constrained. This is not a desirable situation for a number of reasons, the most obvious being that a "solution" may be hard to come by. 
A more compact way to write the above system is as a single vector equation $\mathbf{F}(\mathbf{c}, \mathbf{z})=\mathbf{0}$. For our purposes, if the problem can be solved for $k=2$, then certainly the more general problem can solved by repeated application of the same method (see section 1.3 below). Therefore, rather than consider equations of this last type, we instead consider an equation of the form $\mathbf{G}\left(\mathbf{f}(\mathbf{c}), z_{1}, z_{2}\right)=0$ where $\mathbf{f}$ is a smooth function mapping the space that $\mathbf{c}$ lives in to $\mathbf{R}$ or $\mathbf{C}$. Here we will take $\mathbf{f}$ to be the composition of the averaging operator, $\mathbf{A}$, with various analytic functions that act pointwise on $\mathbf{c}$. We say that $\mathbf{f}(\mathbf{c})$ is a signature of $z_{1}, z_{2}$. The fact that $\mathbf{f}$ is smooth makes the system robust to errors in measurement, while taking advantage of global information that may be embedded in the measured data. A signature is a quantity that can be measured experimentally, with the goal being to find the geometry of the configuration $\left(z_{1}, z_{2}\right)$ from this value.

\subsection{Structure from Motion}

The problem of obtaining the geometry of a scene by moving a camera within it is a well-known one, and has many different forms. If the pose of the camera is known at all times, then reconstructing the geometry of the scene is easier than if the position is an unknown. The latter problem is known as the structure from motion problem. In this problem, the variables representing the poses of the camera are unknown, so one needs to take enough snapshots of the environment in order to solve for these variables plus the variables that represent the geometric structure of the environment. Because measurements from the camera have errors, one usually takes many "extra" snapshots in order to over-constrain the problem.

In this paper we will consider only the $2 \mathrm{D}$ version of the structure from motion problem, and take a very different approach than that described above. We investigate certain signatures associated with moving a camera on a circle and show how they can be used to reconstruct the positions of an unknown points.

\subsection{Problem Statement}

The general form of our problem is the following: Suppose that $z_{1}, \ldots, z_{n}$ are complex numbers representing $n$ landmarks in the plane, and a sensor is available with the ability to measure the angles between the landmarks with respect to any given position of the sensor, $w$ (i.e. it can measure the angle $\angle z_{i} w z_{j}, i, j=$ $1, \ldots, n)$. Then for $m$ positions $w_{1}, \ldots, w_{m}$ of the sensor ${ }^{1}$, can one determine $z_{1}, \ldots, z_{n}$ and $w_{1}, \ldots, w_{m}$ given only the measurements $\angle z_{i} w_{j} z_{k}, i, k=1, \ldots, n, j=$ $1, \ldots, m ?$

One approach to the above is to write down all of the trigonometric equations associated with the configuration. Since the angles are known, the resulting

\footnotetext{
${ }^{1}$ In our model of the panoramic sensor, the pose of the sensor means only its position, and not orientation. For real applications it is possible to define and compute and orientation with our method if it is needed.
} 
polynomial system has many more equations than variables. Thus the system is over-constrained, which may be helpful since in a real experiment the angular measurements will contain noise. Then one may try to find a solution in the "least squares sense" by minimizing the sum of the squares of these equations.

In the problem we address here we will make the assumption that the points $w_{i}$ all lie on a circle i.e. $\left|w_{i}\right|=1, i=1, \ldots, m$, but we will not assume where on the circle the $w_{i}$ are. Additionally, we will assume that the $w_{i}$ are densely distributed in the circle in an approximately uniform fashion. Also, notice that if the problem can be solved for two landmarks then it can be solved for more than two landmarks by applying the same method to pairs of the landmarks.

\subsection{Panoramic Sensors}

Recently, many researchers in the robotics and vision community have begun to investigate the use of curved mirrors to obtain panoramic and omni-directional views. Typically, such systems consist of a camera pointing upward at a convex mirror, as in figure 2. How to interpret and make use of the information obtained

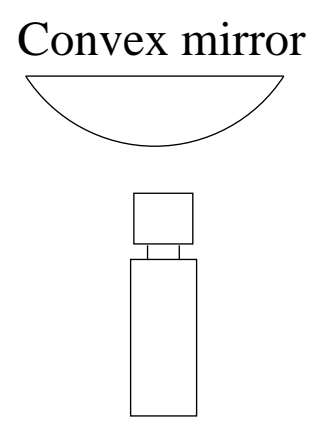

Camera

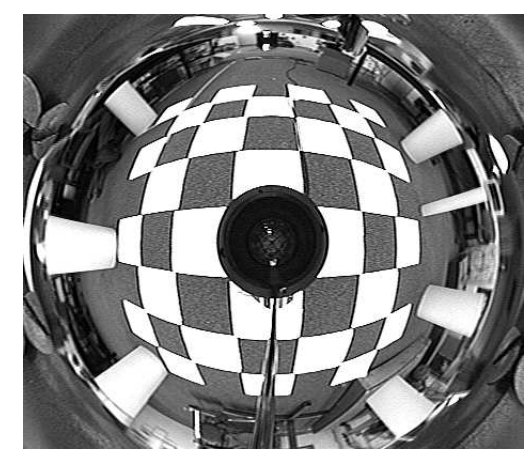

Figure2. On the left we see one way to create a panoramic sensor: point a camera upwards at a curved mirror. On the right we see an image from such a panoramic sensor, with the camera visible in the center.

by such sensors, e.g. how it can be used to control robots, is not immediately clear. It does seem likely that panoramic systems may be able to handle some problems that are difficult or impossible to address with a standard camera. To address our problem experimentally, we employed such a panoramic sensor, using a spherical mirror. A natural feature to consider for extraction from an image taken on such a system is any edge that is vertical in the real world, because these edges appear as radial lines in the image (see figure 2). In the center of this image the lens of the camera is clearly visible. To measure the angles between the vertical edges we extend them to the center of the lens and compute the angle at their intersection. 


\subsection{Contributions}

Our reconstruction method has a number of features that other methods do not.

(1) Rather than constructing one or more equations for each measurement, we first process the measured data to produce two numbers (signatures), which are used to construct two equations in the positions of the unknown landmarks. These equations are quadratic in general and linear if a single reference point is known.

(2) Due to the simplicity of the equations, optimization methods are not needed, and when the solutions are found they are known to be true solutions, as opposed to possible spurious solutions produced by numerical optimization methods.

(3) Due to the stable means of processing the data (integration), our method handles noise well. In addition, if one desired to carry out a detailed error analysis, it would be possible since the equations are so simple. This is not the case with a method that produces a very large number of nonlinear equations.

\subsection{Related Work}

Due to space limitations we briefly summarize here the related work. The precursor to this work is [?], where a similar problem was considered, but explicit solutions were not possible. Regarding the reconstruction aspect of our work, there is work in the eighties - early nineties on reconstructionb using vertical edges and known motion (Kriegman [5], Kak [4]). There is work in structure from motion from circular trajectories (Shariat and Price [9], Sawhney [8]). This work, however, uses a set of equations with the constraint that the projection centers are on a circle.

Recently, a number of approaches to navigation and reconstruction using omnidirectional systems have been proposed (Nayar [6], Svoboda [11], Onoe [7], Srinivasan [2], Yagi [12], Medioni [10]). The work by Yagi and Medioni is very similar but uses an already known environmental map. The most relevant to this paper is the work on omnidirectional multibaseline stereo done by Kang and Szeliski [3], but this work uses conventional cameras and measurement equations.

\section{Calculating Signatures}

\subsection{Angles and Complex Logarithms}

Definition A function is holomorphic on an open subset $U \subset \mathbb{C}$ if it is complex differentiable at every point of $U$.

For any complex number $z=a+b i$, we define the exponential function by $\exp (z)=e^{a}(\cos (b)+i \sin (b))$. The exponential function is a holomorphic function which assumes all complex values except 0 . If $U$ is an open connected subset of $\mathbb{C}$, then a holomorphic function, $\ell$, is called a logarithm function on $U$ if for all $z \in U, \exp (\ell(z))=z$. 
We use $\mathbb{R}^{-}$to denote the set of non-positive real numbers and $\mathbb{C}^{-}$to denote the "slit" complex plane, $\mathbb{C}-\mathbb{R}^{-}$. If $z \in \mathbb{C}^{-}$, then there are unique real numbers $\theta$ and $r$ with $r>0$ and $-\pi<\theta<\pi$, such that $z=r e^{i \theta}$. Given a number, $z$, written in this form, the principle branch of the logarithm, Log, is defined by

$$
\log (z)=\ln (r)+i \theta,
$$

where $\ln$ is the real natural logarithm. It is easy to check that the principle branch of the logarithm is a logarithm in the sense defined above. The set $\mathbb{R}^{-}$ is called the branch cut of the Log function.

It is possible to define logarithm functions other than Log using the above method.For example, we could choose $0<\theta<2 \pi$ and take the branch cut to be the set of non-negative real numbers. Thus we can always construct a logarithm by choosing a ray emanating from the origin to slit the plane along and an open interval of length $2 \pi$ from which the angle is chosen. From this point on, we will use "log" denote a fixed one of these branches of the logarithm, but we won't specify which one unless it is necessary.

Next, observe that $\theta=\Im(\log (z))$ where $\Im(z)$ means the imaginary part of $z$. This allows us to define the (signed) angle between $z_{1}, z_{2} \in \mathbb{C}^{-}$by

$$
\angle\left(z_{1}, z_{2}\right)=\Im\left(\log \left(z_{1}\right)-\log \left(z_{2}\right)\right) .^{2}
$$

\subsection{Averaging Functions of $\Theta$}

We denote the unit circle in the complex plane as $S^{1}=\left\{e^{i t} \mid t \in[0,2 \pi]\right\}$. Let $z_{1}$ and $z_{2}$ be complex numbers. Then we define the angle between $z_{1}$ and $z_{2}$ with respect to a third point $z$ as

$$
\Theta(z)=\Im\left(\log \left(z_{1}-z\right)-\log \left(z_{2}-z\right)\right) .
$$

We emphasize here that our definition of $\Theta$ depends on the choice of logarithm and on $z_{1}$ and $z_{2}$. The domain of $\Theta$ is the complex plane minus two parallel slits, one emanating from $z_{1}$ and the other from $z_{2}$. On these slits, $\Theta$ is, of course, not defined.

The average angle between $z_{1}$ and $z_{2}$ with respect to a point moving on the unit circle can then be represented by the integral of $\Theta$. Our first result is

Theorem 1. If for a given $z_{1}, z_{2}$ and a choice of $\log , \Theta$ is defined on all of $S^{1}$, then

$$
\frac{1}{2 \pi} \oint_{S^{1}} \Theta d t=\angle\left(z_{1}, z_{2}\right)
$$

where the same log function used to define $\Theta$ is also used to define the $\angle$ function.

${ }^{2}$ At this point it is tempting to combine these terms, but unfortunately complex logarithms do not enjoy all of the same properties of real logarithms. 
Proof Viewed as a function of $(x, y), \Theta$ is a harmonic function (i.e. it is a solution to Laplace's equation $\nabla^{2} \Phi=0$ ) since it is the imaginary part of a holomophic function. It is a well known fact that if a harmonic function is defined on a disk then the average value of that function on the boundary of the disk is equal to the value of the function at the center of the disk. By hypothesis, the branch cuts of $\Theta$ do not cross the unit disk, so $\Theta$ is harmonic there and hence its average value on the boundary of disk $\left(S^{1}\right)$ is equal to the value of $\Theta$ at the origin, i.e. $\Im\left(\log \left(z_{1}\right)-\log \left(z_{2}\right)\right)=\angle\left(z_{1}, z_{2}\right) \cdot \diamond$

If, for example, we chose to use the principle logarithm function, Log, then the theorem applies to any $z_{1}$ and $z_{2}$ as long as they do not lie to the left of $S^{1}$.

The above theorem tells us that integrating the angular data that can be measured tells us something about the geometry of $z_{1}$ and $z_{2}$. Given this, it seems likely that integrating a function of $\Theta$ could yield more information about $z_{1}$ and $z_{2}$. (From an experimental point of view, what this means is that the angular data is gathered in the form of a list of angles, and then this list is transformed by the above function and then averaged.) In particular, we would like to determine the magnitudes of $z_{1}$ and $z_{2}, r_{1}$ and $r_{2}$, resp. To do this a more explicit expression for $\Theta$ on $S^{1}$ is desirable. Using the identity $\Im(z)=\frac{z-\bar{z}}{2 i}$ and the fact that $\bar{z}=\frac{1}{z}$ for $z \in S^{1}$, we have that for $z \in S^{1}$

$$
\Theta(z)=\frac{1}{2 i}\left(\log \left(z_{1}-z\right)-\log \left(z_{2}-z\right)-\log \left(\overline{z_{1}}-\frac{1}{z}\right)+\log \left(\overline{z_{2}}-\frac{1}{z}\right)\right) .
$$

It is important to keep in mind that the right hand side of (5) is an expression for $\Theta$ restricted to $S^{1}$, and does not agree with $\Theta$ off of $S^{1}$. In particular, the right hand side of (5) defines a holomorphic function, whereas $\Theta$ is harmonic, but not holomophic, since it is real valued and non-constant.

Given (5), a natural function to integrate would appear to be $e^{2 i \Theta}$. Thus, the quantity we want to compute is (for $z_{1}$ and $z_{2}$ fixed)

$$
\frac{1}{2 \pi} \oint_{S^{1}} e^{2 i \Theta} d t
$$

This integral can be written as a complex contour integral by using the parameterization $z=e^{i t}$, so that $\frac{d z}{d t}=i e^{i t}=i z$. Therefore $d t=\frac{d z}{i z}$ and so (6) is equal to

$$
\frac{1}{2 \pi} \oint_{S^{1}} \frac{\left(z_{1}-z\right)\left(\bar{z}_{2}-\frac{1}{z}\right)}{\left(\bar{z}_{1}-\frac{1}{z}\right)\left(z_{2}-z\right)} \frac{d z}{i z}
$$

The function $g(z)=\frac{\left(z_{1}-z\right)\left(\bar{z}_{2}-\frac{1}{z}\right)}{\left(\bar{z}_{1}-\frac{1}{z}\right)\left(z_{2}-z\right)} \frac{1}{i z}$ has singularities at $0, \frac{1}{\bar{z}_{1}}$ and $z_{2}$, all of which are simple poles. Therefore the residues at these poles can easily be computed:

$$
\operatorname{Res}(g, 0)=\lim _{z \rightarrow 0} z g(z)=-i \frac{z_{1}}{z_{2}}
$$




$$
\begin{aligned}
& \operatorname{Res}\left(g, \frac{1}{\bar{z}_{1}}\right)=\lim _{z \rightarrow \frac{1}{z_{1}}}\left(z-\frac{1}{\bar{z}_{1}}\right) g(z)=\frac{i\left(z_{1} \bar{z}_{1}-1\right)\left(\bar{z}_{1}-z_{2}\right)}{\left(\bar{z}_{1}^{2} z_{2}-\bar{z}_{1}\right)} \\
& \operatorname{Res}\left(g, z_{2}\right)=\lim _{z \rightarrow z_{2}}\left(z-z_{2}\right) g(z)=\frac{i\left(z_{1}-z_{2}\right)\left(z_{2} \bar{z}_{1}-1\right)}{\bar{z}_{1} z_{2}^{2}-z_{2}}
\end{aligned}
$$

We now state the residue theorem (see [1]) for completeness.

The Residue Theorem Let $C$ be a positively oriented simple closed contour within and on which a function $f$ is analytic except at a finite number of singular points $w_{1}, w_{2}, \ldots, w_{n}$ interior to $C$. If $R_{1}, \ldots, R_{n}$ denote the residues of $f$ at those respective points then $\int_{C} f(z) d z=2 \pi i\left(R_{1}+\cdots+R_{n}\right)$.

Thus the value of (7) depends upon whether or not $z_{1}$ and $z_{2}$ lie inside or outside of $S^{1}$. ( $z_{1}$ and $z_{2}$ may not lie on $S^{1}$ for this method to work, and we will not consider this case.) The above calculation immediately gives our main result.

Theorem 2. The value of the integral (6) is

$$
\begin{aligned}
& \text { (a) } \overline{\left(\frac{z_{2}}{z_{1}}\right)} \text { if }\left|z_{1}\right|>1,\left|z_{2}\right|<1 \\
& \text { (b) } \frac{-z_{1} \bar{z}_{1}+z_{2} \bar{z}_{1}-\bar{z}_{2} z_{2}+z_{1} z_{2} \overline{z_{2} z_{1}}}{z_{2}\left(-1+z_{2} \bar{z}_{1}\right) \bar{z}_{1}} \text { if }\left|z_{1}\right|>1,\left|z_{2}\right|>1 \\
& \text { (c) } \frac{z_{1} \bar{z}_{1}-1-z_{1} \bar{z}_{2}+\bar{z}_{2} z_{2}}{-1+z_{2} \bar{z}_{1}} \text { if }\left|z_{1}\right|<1,\left|z_{2}\right|<1 \\
& \text { (d) } \frac{z_{1}}{z_{2}}, \text { if }\left|z_{1}\right|<1,\left|z_{2}\right|>1
\end{aligned}
$$

To see how the above theorem can be applied to the problem stated in the introduction, suppose for example that $z_{2}$ is known and let $A$ (for average) be the experimentally measured value of the integral (6). Then the above theorem tell us that if $\left|z_{1}\right|>1$ and $\left|z_{2}\right|<1$, then $z_{1}=z_{2} \cdot \frac{1}{\bar{A}}$, i.e. we have a linear solution to the reconstruction problem.

Next we demonstrate how to solve the more general problem when $z_{1}$ and $z_{2}$ are both unknown. Suppose that $z_{1}=r_{1} e^{i \Theta_{1}}$ and $z_{2}=r_{1} e^{i \Theta_{2}}$, where $r_{1}, r_{2}>1$, i.e. we are in case (b) of theorem 2. Let $A$ be the value of (6) and let $\psi$ be the angle between $z_{1}$ and $z_{2}$ with respect to the origin, chosen with the orientation such that $z_{2} \bar{z}_{1}=r_{1} r_{2} e^{i \psi}$. Theorem 2 part (a) may be rewritten as

$$
\frac{-r_{1}^{2}+r_{1} r_{2} e^{i \psi}-r_{2}^{2}+r_{1}^{2} r_{2}^{2}}{r_{1} r_{2} e^{i \psi}+r_{1}^{2} r_{2}^{2} e^{2 i \psi}}=A
$$

where $\psi$ may be considered as a known quantity because of theorem 1 . This single equation between complex quantities can be converted to two real equations in $r_{1}$ and $r_{2}$. The easiest way to do this is first make the substitutions $u=r_{1} r_{2}$ and $v=r_{1}^{2}+r_{2}^{2}$ and rationalize. Then (11) becomes $-v+u e^{i \psi}+u^{2}=\left(u e^{i \psi}+u^{2} e^{2 i \psi}\right) A$. Taking the real and imaginary parts of the above equation gives linear equations 
for $u$ and $v$. Once $u$ and $v$ are known, we must determine $r_{1}$ and $r_{2}$ from the equations $u=r_{1} r_{2}$ and $v=r_{1}^{2}+r_{2}^{2}$. These last two equations can clearly be converted to a single quadratic equation in $r_{1}^{2}$, allowing for the solution of $r_{1}$ and $r_{2}$.

\section{Experimental Results}

The main question to be addressed is the effect of noise on the above method when both $z_{1}$ and $z_{2}$ are unknown. The primarily source of error is in the data taken from the panoramic sensor, so we need to know the nature of the noise that we expect from using this device to measure angles. From experiment we have found the error in angular measurement between vertical edges to have a standard deviation, $\sigma$, of about .05 degrees at a range of one meter. To be conservative, in our simulations we varied $\sigma$ from 0 to .2 degrees.
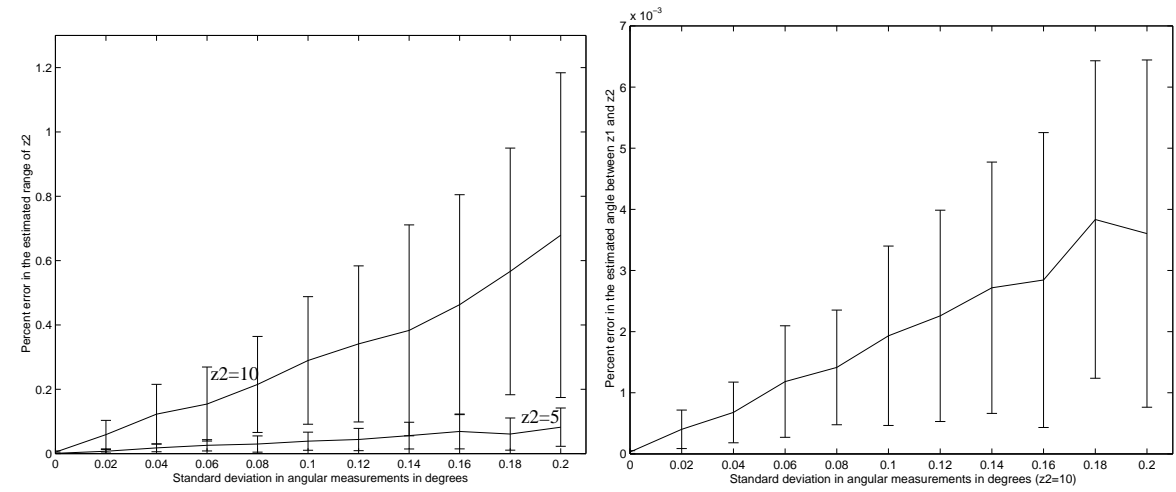

Figure3. Above on the left appears a plot of percent error in estimating the range of $z_{2}$ and versus the standard deviation in the noise (in degrees) of the measured angles, and on the right a similar plot for the estimate in the angle between $z_{1}$ and $z_{2}$. In both plots $z_{1}=5 e^{\frac{i \pi}{3}}$. Note the extremely high accuracy of the estimate of the angle between $z_{1}$ and $z_{2}$

Another source of error is the computation of the averages of $\Theta$ and $e^{2 i \Theta}$. We are approximating an integral with an average, and because we do not require any knowledge of where on the circle the sensor is, the average is only a good approximation if the density of the measurements is approximately uniform. Thus in the simulation we generated points uniformly spaced on the circle and added noise to the angular position of each point. We always took this noise to be zero mean Gaussian noise with standard deviation one-quarter of $2 \pi$ divided by the number of sample points. This value of course depends on the experimental device being modeled.

There are many parameters that can be varied in simulation, such as the magnitude of the points being reconstructed, the number of data points used, 
the numerical integration scheme and so on. Therefore we first fixed the two known points to be $z_{1}=5 e^{\frac{i \pi}{3}}$ and $z_{2}=5$, fixed the number of measurements taken on the circle to be 5000, and considered the effect of zero mean Gaussian noise with $\sigma$ ranging from 0 to .2 degrees. Given all of the above parameters 100 trials were performed and in each trial the percent error in the magnitude of $z_{2}$ was computed and an average over all trials of this error was computed.

To demonstrate the effect of range we then repeated this entire simulation with $z_{2}=10$. Each choice of $z_{2}$ yields a plot of percent error versus the standard deviation in the noise, which both appear in figure 3 .

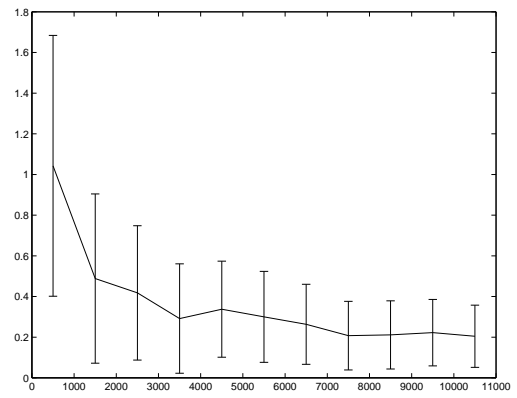

Figure4. A plot of the percent error in the estimated value of the magnitude of $z_{2}$ versus the number of sample points used on the circle. Here we fixed $z_{1}=5 e^{\frac{i \pi}{3}}$ and $z_{2}=10$. The error bars represent the standard deviation in one hundred trials.

Figure 4 illustrates the percent error in reconstruction versus the number of sample points used, i.e. the number of angular pairs measured. In this case we fixed $\sigma=.1$ and let $z_{1}=5 e^{\frac{i \pi}{3}}$ and $z_{2}=10$.

Using a panoramic sensor (consisting of a Sony XC-77 camera and a spherical mirror) on a turntable, we performed an experiment to reconstruct the positions of two vertical edges. The edges were the vertical edges of white paper on a black background placed at $2.8\left(r_{1}\right)$ and $2.7\left(r_{2}\right)$ units from the origin (which is the center of the turntable) with an angle of 22 degrees between them. An discussed above, vertical edges in the world appear as radial in the spherical image (see figure 2). Recall that the unit of measure is the distance from the center of the turntable to the optical axis of the camera, which was $18 \mathrm{~cm}$ (The roughness of the measurements is due to the fact that the true values for the distances were found by hand using a ruler.). We then gathered 71 data points by rotating the turntable by hand. The estimates obtained were 2.7 units for $r_{1}$ and 2.7 units for $r_{2}$.

\section{Conclusion}

In this paper we have considered the use of controlled motion for the problem of recovering robot pose and the lay-out of the environment. To do this we have 
introduced certain spaciotemporal signatures that give rise to quadratic or linear equations for the solution of the problem. This is achieved by the application of the residue calculus from the theory of complex variables. The method we propose is robust to noise and outliers and takes into account global information that alternative methods do not.

\section{References}

1. C. Berenstein and R. Gay. Complex variables : an introduction. Springer-Verlag: New York, 1991.

2. J.S. Chahl and M.V. Srinivasan. Range estimation with a panoramic sensor. $J$. Optical Soc. Amer. A, 14:2144-2152, 1997.

3. S. Kang and R. Szeliski. 3-d scene data recovery using omnidirectional multibaseline stereo. International Journal of Computer Vision, 25:167-183., 1997.

4. A. Kosaka and A.C. Kak. Fast vision-guided mobile robot navigation using modelbased reasoning and prediciton of uncertainties. Computer Vision Image Understanding, 56:271-329, 1992.

5. D.J. Kriegman, E. Triendl, and T.O. Binford. Stereo vision and navigation in buildings for mobile robots. Trans. on Robotics and Automation, 5:792-804, 1989.

6. S. Nayar. Catadioptric omnidirectional camera. In Proc. Computer Vision Pattern Recognition, pages 482-488, 1997.

7. Y. Onoe, H. Yokoya, and K. Yamazawa. Visual surveillance and monitoring system using an omnidrectional system. In Proc. Int. Conf. on Pattern Recognition, 1998.

8. H.S. Sawhney, J. Oliensis, and A.R. Hanson. Description and reconstruction from image trajectories of rotational motion. In Int. Conf. computer Vision, pages 494498, 1990.

9. H. Shariat and K.E. Price. Motion estimation with more then two frames. IEEE Trans. Patt. Anal. Mach. Intell., 12:417-434, 1990.

10. F. Stein and G. Medioni. Map-based localization using the panoramic horizon. Trans. on Robotics and Automation, 11:892-896, 1995.

11. T. Svoboda, T. Padjla, and V. Hlavac. Epipolar geometry for panoramic cameras. In Proc. European Conference on Computer Vision, 1998.

12. Y. Yagi, S. Nishizawa, and S. Tsuji. Map-based navigation for a mobile robot with omnidirectional image senso. Trans. on Robotics and Automation, 11:634-648, 1995. 